\title{
Nuclear Power Fault Diagnostics and Preventative Maintenance Optimization
}

\section{January 2021}

Cody Walker, Vivek Agarwal, Nancy Lybeck

Idaho National Laboratory

Pradeep Ramuhalli

Oak Ridge National Laboratory

Mike Taylor

Electric Power Research Institute

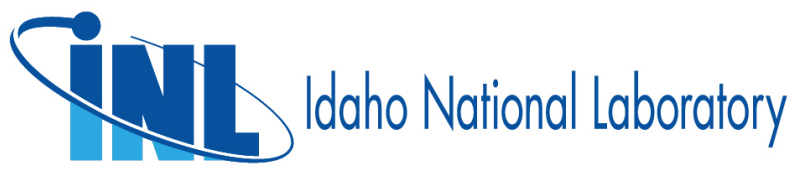

INL is a U.S. Department of Energy National Laboratory

operated by Batelle Energy Alliance, LLC 


\section{DISCLAIMER}

This information was prepared as an account of work sponsored by an agency of the U.S. Government. Neither the U.S. Government nor any agency thereof, nor any of their employees, makes any warranty, expressed or implied, or assumes any legal liability or responsibility for the accuracy, completeness, or usefulness, of any information, apparatus, product, or process disclosed, or represents that its use would not infringe privately owned rights. References herein to any specific commercial product, process, or service by trade name, trade mark, manufacturer, or otherwise, does not necessarily constitute or imply its endorsement, recommendation, or favoring by the U.S. Government or any agency thereof. The views and opinions of authors expressed herein do not necessarily state or reflect those of the U.S. Government or any agency thereof. 


\title{
Nuclear Power Fault Diagnostics and Preventative Maintenance Optimization
}

\author{
Cody Walker, Vivek Agarwal, Nancy Lybeck \\ Idaho National Laboratory \\ Pradeep Ramuhalli \\ Oak Ridge National Laboratory
}

January 2021

Idaho National Laboratory
Idaho Falls, Idaho 83415

http://www.inl.gov

Prepared for the U.S. Department of Energy Office of Nuclear Energy Under DOE Idaho Operations Office

Contract DE-AC07-05ID14517 
Page intentionally left blank 


\begin{abstract}
Operation and Maintenance (O\&M) costs for nuclear power plants (NPPs) are very large. The nuclear industry is beginning to see reactors shut down-even after their operating licenses have been extended-because they are not economically competitive with other energy sources. These early closures happen due to economic reasons, despite excellent safety records. Therefore, it is imperative to reduce costs in order to prevent these early closures. This paper showcases recent research on advanced fault diagnostics techniques and preventative maintenance optimization (PMO) for reducing NPP maintenance costs. This report focuses on the feedwater and condensate system (FWCS) for both pressurized- and boiling-water reactor (BWR) systems. The computerized maintenance management system (CMMS), which contains the plant's digital record of all corrective maintenance $(\mathrm{CM})$ and preventative maintenance (PM) work orders, provided the ground truth for locating potential faults and labeling the process data as either healthy or faulted. Various feature extraction techniques were utilized to further differentiate the faulted data from the healthy data. Through a cross-validation procedure, support vectors machines (SVMs) were used to label other test sets of process data as either healthy or faulted. Similar faults were not found within the BWR system, thus opening up the potential for PMO, since an unnecessary amount of PM leads to inflated maintenance costs. This paper summarizes the steps for PMO, from component health determinations to recommendations for action. An example of PMO assessment is presented for condensate pumps (CPs), condensate booster pumps (CBPs), and the respective motors that drive them.
\end{abstract}


Page intentionally left blank 


\section{CONTENTS}

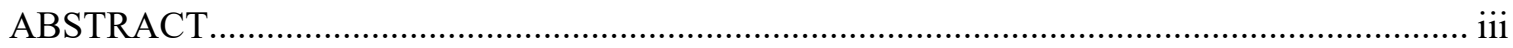

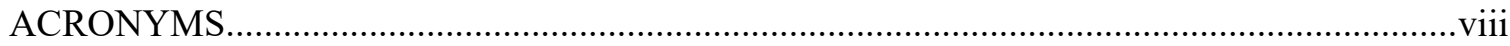

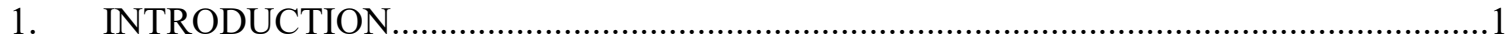

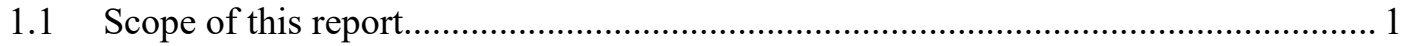

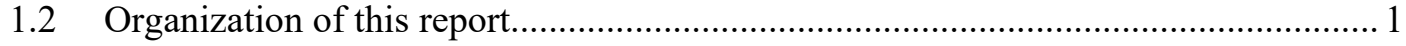

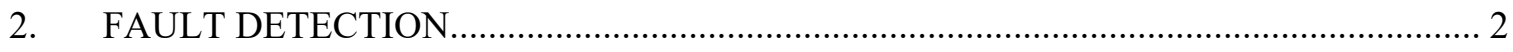

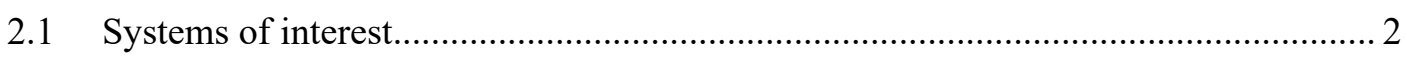

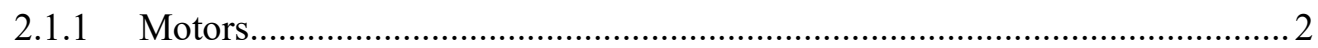

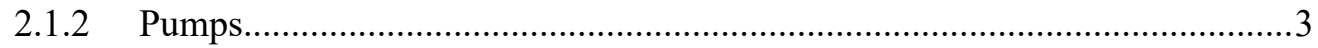

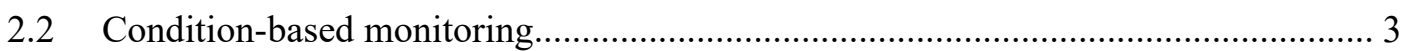

3. PREVENTATIVE MAINTENANCE OPTIMIZATION ...................................................

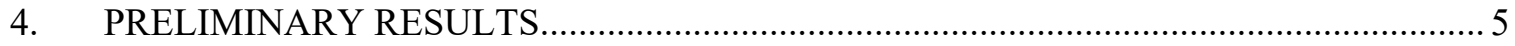

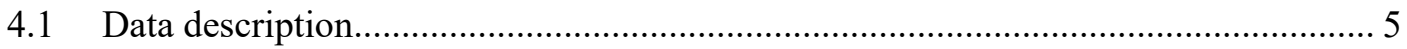

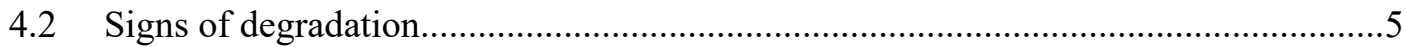

4.3 Preventative maintenance recommendations........................................................ 9

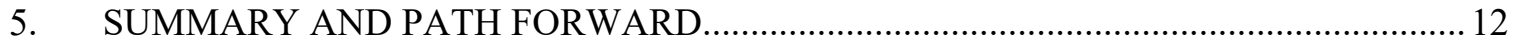

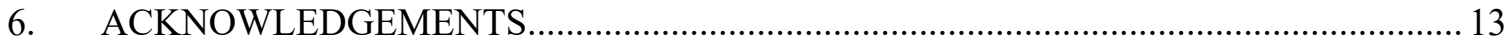

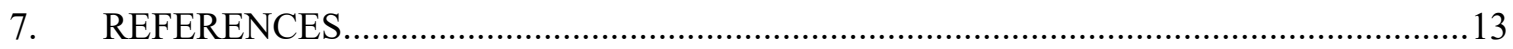

\section{FIGURES}

Figure 1. The average seasonal component temperature was subtracted from the current component temperature. Positive values indicate that the component was hotter than the seasonal average. Highlighted in red is the the section of training data labeled as faulted.

Figure 2. PC analysis was implemented as a preprocessing step to further separate the faulted and healthy datasets.

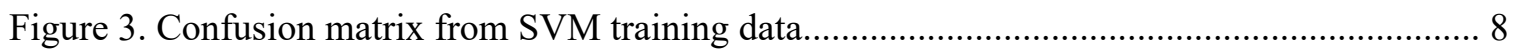

Figure 4. The SVM was used to predict faults in other years. The red spikes indicate either potential faults or inaccurate labels....

\section{TABLES}

Table 1. Example of current PM frequencies and recommendation results

Table 2. Most recent refurbishment work order for the unit $1 \mathrm{CPs}, \mathrm{CBPs}$, and their associated drive motors 
Table 3. Most recent refurbishment work order for the unit 2 CPs, CBPs, and their associated

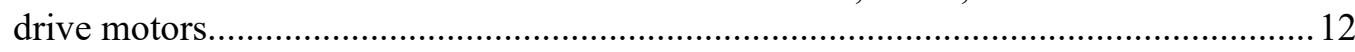


Page intentionally left blank 


\section{ACRONYMS}

$\begin{array}{ll}\text { BWR } & \text { boiling-water reactor } \\ \text { CBP } & \text { condensate booster pumps } \\ \text { CBM } & \text { condition-based maintenance } \\ \text { CM } & \text { corrective maintenance } \\ \text { CLOP } & \text { condensate lube oil pump } \\ \text { CMMS } & \text { computerized maintenance management system } \\ \text { CP } & \text { condensate pumps } \\ \text { EPRI } & \text { Electric Power Research Institute } \\ \text { FWCS } & \text { feedwater and condensate system } \\ \text { ML } & \text { machine learning } \\ \text { NPP } & \text { nuclear power plant } \\ \text { O\&M } & \text { Operations and Maintenance } \\ \text { PC } & \text { primary component } \\ \text { PM } & \text { preventative maintenance } \\ \text { PMBD } & \text { Preventive Maintenance Basis Database } \\ \text { PMO } & \text { preventative maintenance optimization } \\ \text { PWR } & \text { pressurized-water reactor } \\ \text { RPV } & \text { reactor pressure vessel } \\ \text { SVM } & \text { support vector machine } \\ & \end{array}$


Page intentionally left blank 


\section{INTRODUCTION}

For economic reasons, the nuclear industry is witnessing premature closure of nuclear power plants (NPPs), despite excellent safety records [1]. Operations and Maintenance (O\&M) activities are some of the largest costs in operating legacy light-water plants [2]. By reducing O\&M costs, nuclear energy can become more economically competitive with other energy sources. This can be achieved by leveraging machine-learning (ML) and artificial intelligence technologies to develop data-driven algorithms to better diagnose potential faults within the system [3]. Improved accuracy of the models can lead to a reduction in unnecessary maintenance, thus reducing costs associated with parts, labor, and unnecessary planned, forced, or extended outages.

The nuclear industry's excellent safety record is partly due to the amount of preventative maintenance (PM) it performs. Inspections, replacements, refurbishments, vibration monitoring, oil sampling, and other such tasks are all performed at regular intervals [4]. Each task carries an associated cost but reduces the likelihood of certain component failure modes. The frequencies at which these tasks are performed should be reassessed throughout the life of the plant as a means to reduce O\&M costs while still maintaining safety.

\subsection{Scope of this report}

The overall goal of this report is to reduce the costs associated with the nuclear industry's maintenance practices. This is accomplished via a two-prong approach: condition-based monitoring (CBM) and PM optimization (PMO).

First, this report investigates CBM techniques in order to identify potential faults and degradation within the feedwater and condensate system (FWCS)'s condensate pumps (CPs), condensate booster pumps (CBPs), and their associated motors. This report focuses on utilizing heterogenous data in combination with information from the computerized maintenance management system (CMMS) in order to identify potential condition indicators. These indicators were then used in conjunction with ML techniques to seek out other faults within the system. By accurately predicting degradation, the appropriate PM can be scheduled in order to avoid unplanned downtimes and asset failures. Actual plant data from a boiling-water reactor (BWR) FWCS over a large period of time was used in this CBM analysis.

Next, this report examines the application of PMO to update PM frequencies, based on component histories and the opinion of subject matter experts. This research focuses on how to determine whether a component is a PMO candidate, along with the steps to perform the PMO so that labor costs can be minimized by reducing PM frequencies in an intelligent manner. CBM and PM work orders from a pressurized-water reactor (PWR) over a 5-year period were used in the PMO analysis.

This report is the last in a series of documents detailing online monitoring, wireless communication networks, and the development of diagnostic and prognostic models using heterogenous data regarding critical balance of plant equipment in NPPs [5-9]. The previous documents in this series evaluated potential wireless technologies and communication networks inside NPPs, based on their performance and economics [5-7]; assessed vibration sensors with wireless capabilities [8]; and developed formal methodologies for cleaning data and objectively comparing ML prognostic models [9].

\subsection{Organization of this report}

Section 2 details fault detection procedures, including a BWR and PWR FWCS description and CBM overview. Section 3 summarizes the steps necessary for PMO. Section 4 covers the preliminary results from the fault detection algorithms and PMO, and describes the types of data being employed. Section 5 summarizes the report and details both ongoing and future work. 


\section{FAULT DETECTION}

Faults within a system can accumulate into asset failure. Failure does not necessarily mean that the component is physically broken, just that it may not be performing within the desired ranges. Fault detection is the process of detecting deviations from healthy operating conditions, and fault diagnosis classifies the reason behind such deviations. With appropriate sensor data, anomalies can be detected so that appropriate maintenance can be scheduled to remedy the fault [10]. This report focuses on CPs, CBPs, and their associated motors. Data and CMMS work orders were analyzed for both a PWR and BWR system over the last five years.

\subsection{Systems of interest}

The system of interest here is the FWCS. For BWRs, this system supplies the reactor pressure vessel (RPV) with clean, demineralized water at the desired temperature and pressure, in accordance with the rate of steam production. This process begins at the main condenser, which collects a mixture of water and vapor from the turbines. The main condenser condenses the remaining vapor into water, retains the water long enough for the short-lived radioisotopes to decay, and de-aerates the condensate of fission gases. The water is then driven by several CPs, which serve as the driving force for the system. The water then heads through a system of prefilters, demineralizers, and chemical additives that work to condition the coolant. CBPs then provide the motive force to push the condensate through the low-pressure heaters, preheating the coolant before it enters the RPV. This increases the plant's thermal efficiency.

For PWRs, the FWCS provides coolant to the steam generator at the desired temperature and pressure, in accordance with the rate of steam production. PWRs are comprised of two loops. The primary loop provides coolant through the core, while the secondary loop provides water through the steam generator side. The PWR FWCS follows a similar path as the BWR FWCS, but with less conditioning for the condensate, as it does not travel to and from the RPV. The condensate travels from the condenser to the CPs, low-pressure heaters, CBPs, high-pressure heaters, and steam generator.

This study focuses on the CPs, CBPs, and the respective motors that drive them in both BWR and PWR plants. In BWR plants, the condensate motor drives a pair of CPs and CBPs. In PWR plants, each $\mathrm{CP}$ and $\mathrm{CBP}$ has its own respective motor driving it. Specifics on each of these components are given below.

\subsubsection{Motors}

A BWR FWCS is propelled by four sets of CP and CBP pairs. Each pair of pumps is driven by a common motor. This motor is a 200 -amp, 1750 -hp, horizontally positioned, oil-lubricated induction motor. The PWR FWCS is propelled by three sets of CPs and CBPs. Each pump is driven by its own motor. The PWR CP motors are vertical 1250-hp motors. The PWR CBP motors are horizontal 2000-hp motors.

These motors convert electrical energy into torque, and potential failures can be found throughout this process. Common failure locations include the frame and mounting, electrical leads, stator windings, shaft, bearings, rotor, and lubricant [11]. Failures within these locations can be found via the appropriate discovery method. Such discovery methods include inspections, oil analysis, electrical tests, bearing temperatures, ultrasonic monitoring, pressure tests, winding resistance, thermography, etc. [12]. No single method can catch every form of degradation, so a suite of methods must be employed. Inspections can uncover most failure types but are often expensive, time-consuming, or require the system to be offline. By utilizing the appropriate sensors that collect data from heterogenous sources, both common and severe failure mechanisms should be identifiable. 
PM aims to reduce the likelihood of failure through routine tasks. PM tasks for the motors include inspections, electrical testing, vibration analysis, and refurbishments [12]. Inspections aim to discover potential problems with baffles, bearing seals, insulation, mounting, gaskets, motor leads, cages, shafts, stator windings, etc. Electrical testing shows problems with the electrical connections, capacitors, windings, and lamination. Vibration analysis can provide insight into faults stemming from rotating machinery, including bearings, mounting, shafts, and rotors. Refurbishments, sometimes called "overhauls," aim to repair motors into good-as-new condition.

\subsubsection{Pumps}

The CPs and CBPs within the BWR FWCS are horizonal, single-stage, centrifugal-type pumps. They can move water at a rate of $6,825 \mathrm{gal} / \mathrm{min}$. All four are required for normal full-load operation. Each $\mathrm{CP}$ is connected by a common motor to the CBP. In the PWR FWCS, the CPs are vertical, three-stage centrifugal pumps, while the CBPs are horizontal, single-stage centrifugal pumps. The water leaving the condenser is at low pressure and $90 \mathrm{~F}$. The FWCS works to raise these readings to $1,100 \mathrm{psi}$ and $425^{\circ} \mathrm{F}$.

The centrifugal pump uses the motor's rotational energy to increase the flow rate and pressure of the water within the system. Common failure locations for centrifugal pumps include seals, bearings, connections, and gaskets, as well as the impeller, casing, lubricant mounting, shaft, and stuffing box. PM tasks for the pumps are similar to those for the motors, except in regard to electrical testing.

\subsection{Condition-based monitoring}

CBM emphasizes the condition monitoring aspect of maintenance. Data and inspections are used in combination to infer the health or status of the component. Once a sign of degradation is found and the degradation can be diagnosed, the damage can then be trended. Maintenance can be scheduled and performed prior to the component failing to meet its performance criteria.

Faults can be detected using data-driven or model-based techniques [13]. Data-driven techniques utilize sensor data collected from the system and do not require expert knowledge of the physics associated with the system. Data-driven techniques require a sufficient amount of historical data regarding system operations under various conditions. For the models to be most effective, the training data should also include possible failure events. Such data can be difficult to obtain for NPP components, since safety is such a key priority. Fault detection in this case may appear similar to anomaly detection, due to the ratio of healthy to faulted data collected. Some data-driven ML techniques include artificial neural networks [14], auto-associative kernel regression [15], and support vector machines (SVMs) [16-19]. This report focuses on the capability of SVMs to separate healthy and faulted sensor data.

SVMs are classification algorithms that aim to create a hyperplane to separate out two sets of data: healthy and faulted. The core concepts of SVMs are the kernel function and optimization routine $[16,17]$. In most cases, the two sets of data will not be linearly separatable, so the input data must be transformed to a higher-dimensional space via a kernel function. The kernel function returns the inner product between two points for a particular feature space. Common kernel functions include the polynomial, sigmoid, and radial basis functions [18]. The equation for the radial basis function is given in Equation 1.

Once the data has been transformed into a higher-dimensional space, a hyperplane must be created to separate the two datasets. An optimization routine is used to find the hyperplane that best separates the datasets by maximizing the margin between the two classes. The fitted hyperplane is described by the data points closest to the margin's boundary, which are then called "support vectors." These support vectors can be used to classify new sets of data. SVMs are noted for their accuracy, regularization, and generalization abilities [19].

SVM is a supervised learning technique, meaning it requires labeled training data. In practice, such data can be difficult to obtain. For this report, labeled data is obtained by combining the CMMS with the opinion of subject matter experts. The CMMS catalogues all maintenance actions performed on the 
system. This includes maintenance to fix faults (e.g., oil leaks) and conduct bearing replacements. Before the system is taken offline for corrective maintenance $(\mathrm{CM})$, the system is assumed to be operating in a degraded state that will be labeled as faulted. Exactly when this degraded state began is determined via the opinion of subject matter experts, based on deviations in temperature measurements from expected conditions. In many mechanical applications, a rise in temperature correlates with degraded performance, typically through increased vibration or friction.

Model-based techniques require a priori knowledge about the system and its potential failures [3]. These models can be developed based on first principles such as Newton's laws, but require detailed information about the materials used, the failure mechanisms, and the operating conditions. These types of models can be difficult and costly to create. Model-based techniques are outside the scope of this paper.

\section{PREVENTATIVE MAINTENANCE OPTIMIZATION}

PM is any regularly performed task designed to reduce the likelihood of failure. This includes tasks such as component refurbishments, electrical testing, lubrications, cleanings, and replacements. Each of these tasks can reduce the likelihood of certain failure modes. Oil and vibration analyses, operator rounds, and thermography are all examples of the types of monitoring that provide indications of degradation. They can be used to determine and predict the condition of components. CBM activities can be scheduled and performed in a timely manner prior to any in-service failure. The initial PM frequencies were established through a combination of vendor recommendations, expert opinions, and operating experience. As more experience is obtained, these frequencies should be re-examined and then adjusted accordingly. Poor performance may require more PM in order to reduce in-service failures, whereas excellent performance may suggest that less PM is required, thus leading to reductions in overall O\&M costs. PMO should take into account expert knowledge and the historical health of the component when creating a basis for determining which PM frequencies can be extended. This report focuses on an analytical approach for determining which PM task frequencies to extend.

First, the historical health of the component is analyzed to determine whether the component is eligible for PMO. In this report, the components' health was determined from the CM work orders found in the CMMS. The CM work orders detail every component-associated action that is not a regularly scheduled activity. Additional vibration measurements, replacements, fixes, and performance verification are all activities found in CM work orders. Leaks, repairs, and replacements indicate that degraded performance has occurred within the component. If said events occurred recently, the associated components would be ineligible for PMO. However, PM is not capable of preventing all faults. The goal of PMO is to optimize resources in order to maximize safety and uptime while also reducing costs. If a component is only experiencing one type of fault, the frequencies of PM unrelated to that particular failure mode can still be potentially extended as well. For example, if the nuisance fault is produced by leaking seals, that is something discovered primarily through inspections. Oil and vibration analyses are unrelated to seal leakage, so the opportunity to extend their PM frequencies may still apply.

Once historical data have been used to identify a component as healthy, each PM task and the frequency thereof should be evaluated against industry averages or an expert database such as the Electric Power Research Institute (EPRI)'s Preventive Maintenance Basis Database (PMBD). The EPRI PMBD is a maintenance information database containing recommendations on PM frequencies for major components related to power generating facilities. A justification for extending certain PM frequencies can be made by comparing current PM frequencies against those recommendations found in the EPRI PMBD. The PMO process is a delay feedback loop. Effects from extending the PM frequencies will not be seen immediately, so extending them should consist of small, iterative steps. 


\section{PRELIMINARY RESULTS}

This section provides an overview of the sensor data and CMMSs from two different types of operational NPPs. The primary objective of this analysis is to find potential faults within the FWCS and to assess the historical health of each component. If the assessment reveals the component to be healthy, PMO will be applied where possible. By reducing PM frequencies in an intelligent manner, maintenance costs can be reduced while still maintaining a reasonable safety margin.

\subsection{Data description}

Different amounts of data were available for the BWR and PWR systems. For the BWR system, sensor data and CBM records were provided for a 5-year period. No PM records were available, so a final PMO cannot be made. However, an assessment of whether PMO is applicable can be made using the data and records available. For the PWR system, a year's worth of sensor data was available for two units, while CM and PM records were available for a 5-year period. This combination is better-suited for PMO, since all the maintenance tasks are available. This dataset may be less suitable for fault diagnostics, depending on the signal of interest. Some temperature signals experience a clear seasonal trend: colder in winter, warmer in summer.

The available sensor data from both systems are similar and include variables such as generator gross load, average feedwater flows, and data on temperatures/pressures for the condensers, CPs, CBPs, and their associated motors for multiple pump trains. Each dataset consists of unlabeled data and is sampled hourly. There was no indication as to whether any portion of the data corresponded with equipment failure. The available datasets each encompassed steady-state operation, shutdowns for refueling, and derates of varying sizes. There was insufficient information to determine the cause of each derate.

Work orders from the CMMS were provided for a 5-year period. For the BWR system, the available work orders included CM work orders - but not PM work orders - for the CPs and CBPs. These work orders contained information on additional vibration testing, reworks, and replacements. For the PWR system, both CM and PM work orders were available. The PM work orders can include information on such things as routine vibration monitoring, refurbishments, and oil sampling. The historical health of the component can be determined and the PM optimized.

\subsection{Signs of degradation}

In this report, fault diagnostics are analyzed from two perspectives: a data-driven perspective and a CMMS perspective. In the data-driven perspective, the sensor data is analyzed for faults, anomalies, or trends that may indicate degradation or reduced performance. The CMMS is then used to provide the ground truth to verify that a fault occurred, based on any CM work orders remedying the issue. Some changes seen within the FWCS sensor data are not due to faults, but rather changes in operating conditions related to other systems. Diagnosing this behavior is difficult, as sensor data from these other systems were unavailable for this report.

The fault diagnostics process from the CMMS perspective begins by analyzing the CMMS for CM work orders related to failures and fixes. The sensor data collected prior to the work order being issued are analyzed for signs of incipient degradation or developing trends over a period of time. It should be noted that not all failure modes can be detected using the sensor data available. Types of available sensor data include gross load, bearing temperatures, pressures, flow, and motor current. Failures concerning seals, shafts, impellers, and oil require some combination of inspection, vibration, and oil analyses. NPPs perform these tasks at regular intervals, but such data were unavailable for this report. Instead, this report focuses on the process data acquired from in-situ sensors. 
The BWR dataset was first analyzed from a data-driven perspective. The dataset was cleaned, and variables were analyzed individually to observe characteristic features from each signal. Some temperature variables associated with the pumps exhibited seasonal trends: hotter in summer, cooler in winter. Since the dataset covered a period of several years, an expected temperature was calculated for each date, using the average temperature calculated from the other years. These averages were then smoothed using a median filter to remove fluctuations in temperatures due to daily or weekly weather variations. The condition indicator for the pump would then measure the difference between the seasonal average and the current component temperature.

The temperature condition indicator measuring the difference between the component's current temperature and the seasonal average can be seen in Figure 1. Positive values indicate that the component is running hotter than the seasonal average; negative values indicate that the component is running cooler. Negative values are dismissed in this analysis because components typically run hotter when certain degradation modes have occurred, due to increased friction. Positive values above a 6-degree Fahrenheit temperature difference were labeled as faulted. This cutoff was chosen based on the component's history before the outage. Other methods, such as step detection, could be deployed to determine deviations in normal operation. The faulted section can be seen in the red highlighted area in Figure 1. The period of time immediately following the faulted section, and for which no data were collected, represents a regularly scheduled refueling outage.

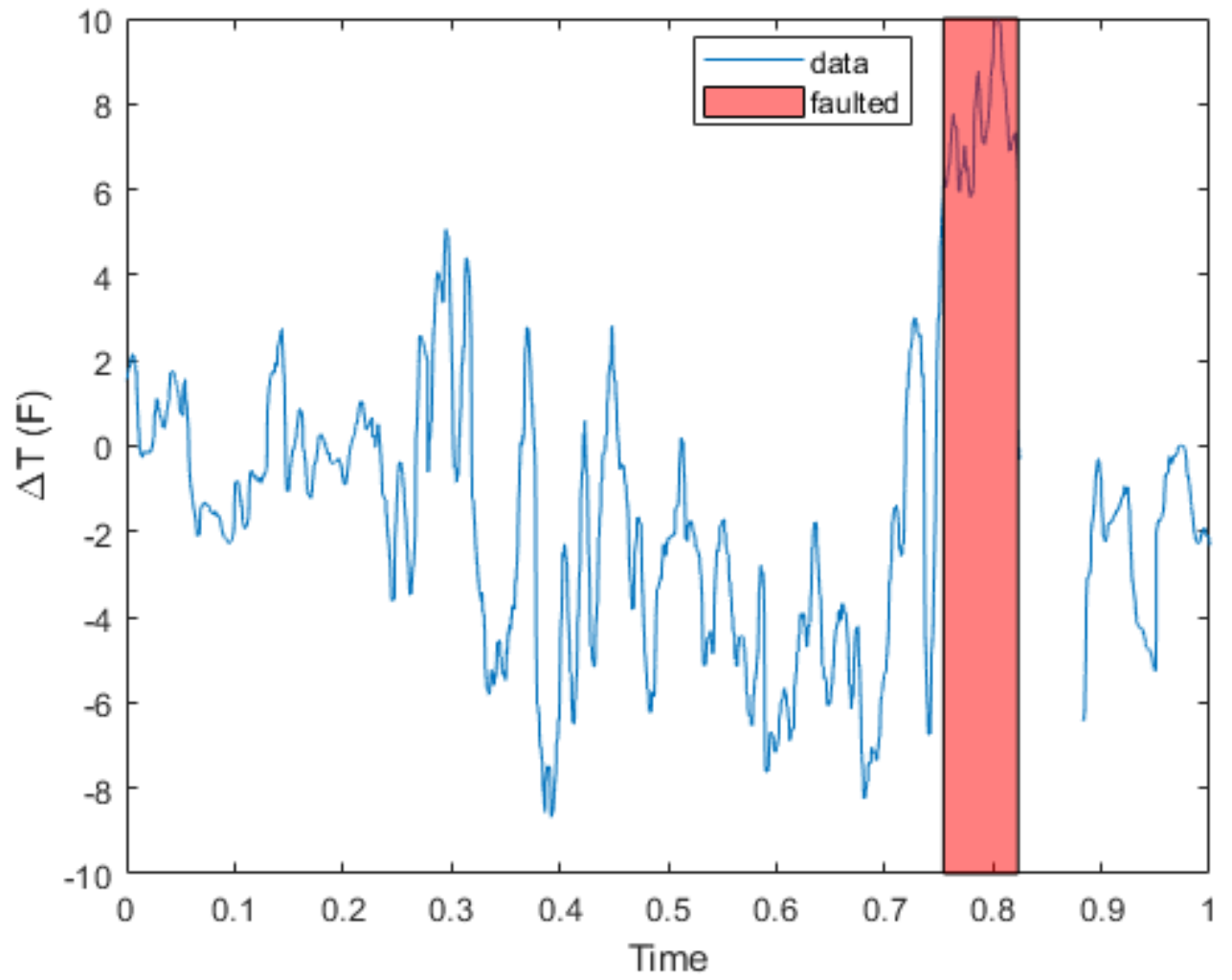

Figure 1. The average seasonal component temperature was subtracted from the current component temperature. Positive values indicate that the component was hotter than the seasonal average. Highlighted in red is the section of training data labeled as faulted.

The temperature condition indicator was combined with other process variables (e.g., gross load, flow, and pressure) to observe the total system response to this potential fault. The high dimensionality of this combination was reduced using principal component (PC) analysis. With just three PCs, $87 \%$ of the information within the dataset was represented. These three PCs are seen in Figure 2. Data within the 
faulted section of Figure 1 are also labeled as faulted in Figure 2, while all other data points are labeled as healthy. Temperature data was not considered when the pump was offline. Figure 2 shows a clear separation between the faulted and healthy data. However, this separation may not be due to the existence of a fault, but rather the ramp down for a planned outage. The temperature difference between the expected and actual temperature values changes in relation to the plant's other process variables. This nonstationary behavior makes it difficult to determine the cause of the separation.

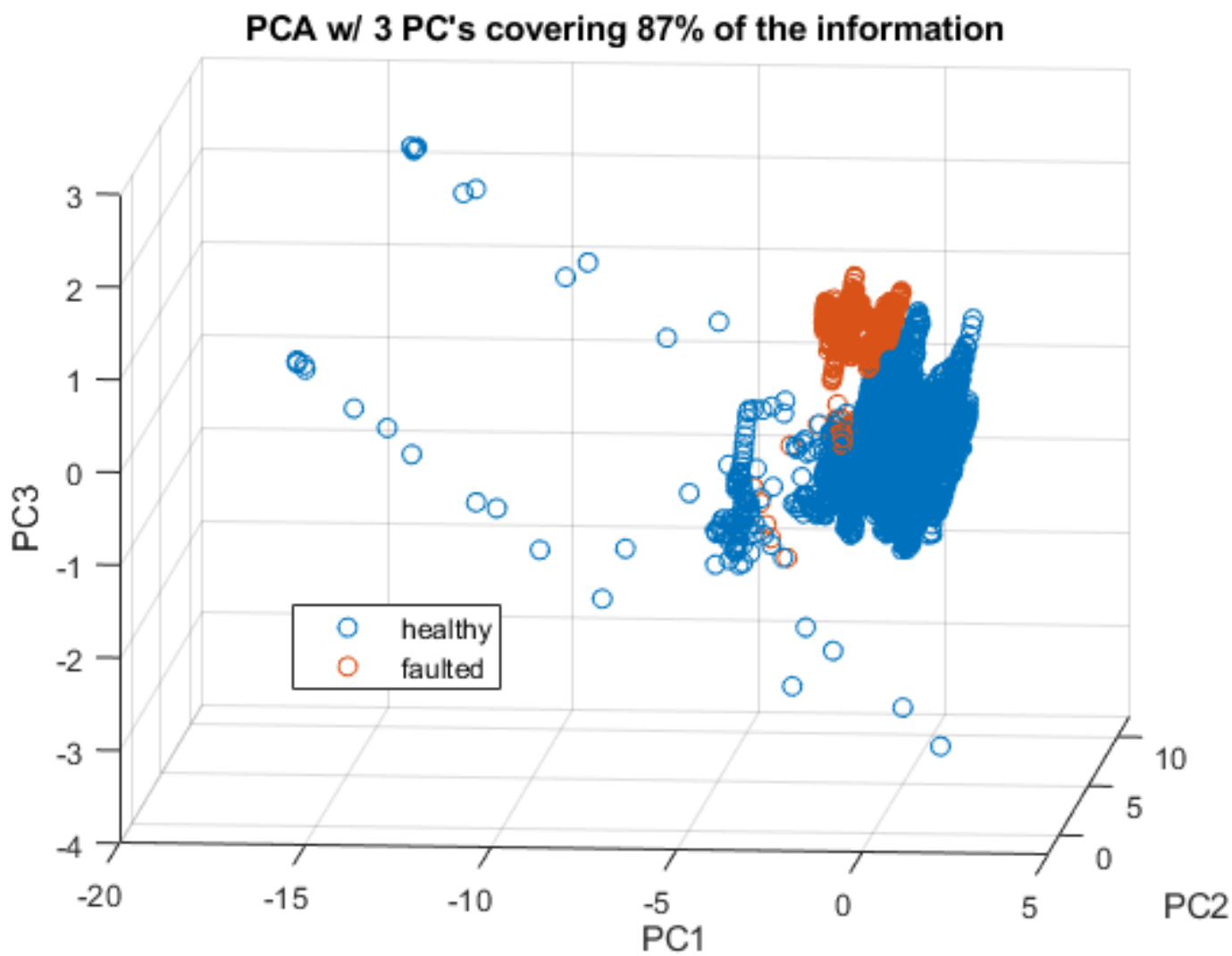

Figure 2. PC analysis was implemented as a preprocessing step to further separate the faulted and healthy datasets.

SVM uses the fault labels to search for similar faults in other years. First, to capture the differences between the healthy and faulted data, the SVM was trained on the data presented in Figure 2. Faulted data made up $4.1 \%$ of the total training data. The SVM was trained using a linear kernel function, resulting in 242 support vectors. The SVM was then tested on the training data to double-check that it could correctly label that data. On the training data, it proved $99 \%$ accurate. The confusion matrix for the training data can be seen in Figure 3, and shows how the model classified each faulted and healthy data point. The SVM was then used to search for similar faults from other years. The test data was normalized and transformed into the same PC space as the training data. The SVM then labeled the test data as either faulted or healthy, as shown in Figure 4. 


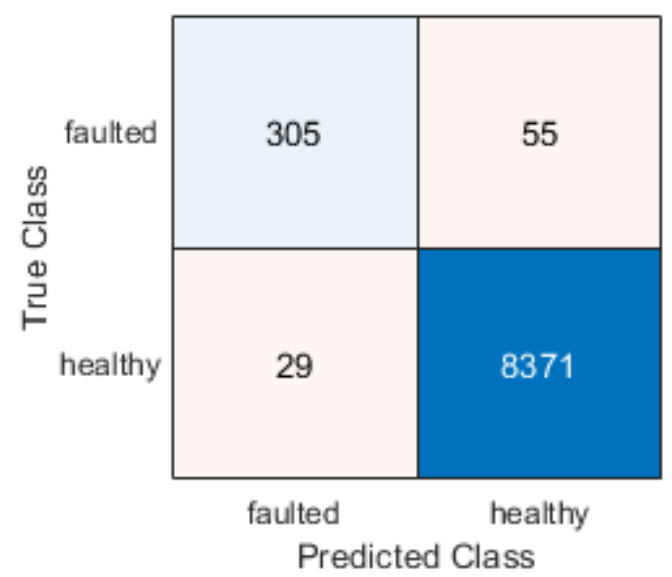

Figure 3. Confusion matrix from SVM training data.

The red spikes throughout Figure 3 show each data point that the SVM labeled as faulted. This test year is interesting because no repairs were made to the CP of interest, and a similar de-ramp into an outage was performed. Since no repairs were made to this pump, all data throughout the test year should be labeled as healthy. Under this assumption, the accuracy of the predictions seen in Figure 4 was $81.4 \%$. The accuracy in predicting other years reached as high as $99.7 \%$ for years involving only healthy, steadystate operation. This high accuracy can seem misleading, but the system was operating under healthy, steady-state conditions over that entire year, so no faults were expected to be identified.

After the data-driven approach was completed, maintenance work orders were reviewed to confirm the presence of a fault. Work orders completed during the outage immediately following the labeled faults seen in Figure 1 suggest that the thermocouple experienced a drift or fault. 


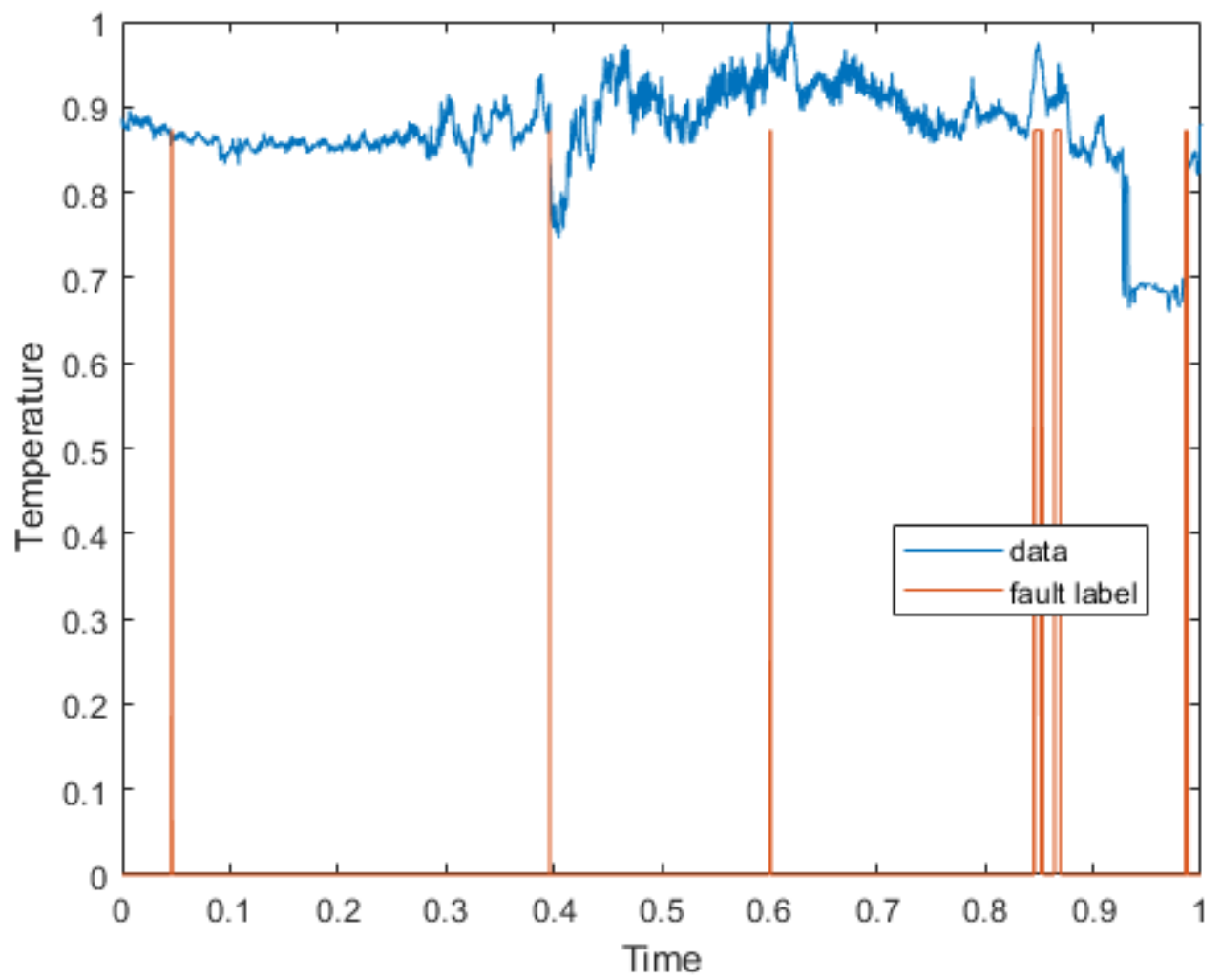

Figure 4. The SVM was used to predict faults in other years. The red spikes indicate either potential faults or inaccurate labels.

Fault diagnostics were then analyzed from the CMMS perspective by reviewing the CM work orders related to failures and fixes. CM work orders generated outside the regularly scheduled refueling outages were given higher priority in this review, since they can immediately affect component uptimes and plant output. One such fault was corroborated using the CMMS. An inboard oil leak was discovered in one of the CPs. This leak was due to a bearing's outer edge riding against the coupling side of the housing. The $\mathrm{CP}$ was taken offline during plant operation to fix the bearing's leak, causing a $27.5 \%$ derate in the plant's output over a 9-hour period.

This leak was most likely discovered during a routine walk-through or via route-based vibration monitoring. The leak was not significant enough to impact those parameters that were being monitored and to which we had access. These parameters included gross load, inboard/outboard bearing temperatures, flow, and pressure. This shows that complete situational awareness is unavailable via the current capabilities of the embedded sensors, causing inspections to remain necessary. A potential way of diagnosing this type of leakage is through the position of the temperature control valve. The temperature control valve position may vary, as the leakage results in less volume to control. Alternatively, the bearing leakage could potentially be discovered via in situ vibration monitoring. Bearing vibrations may be affected by the size and position of the leak, or by how the bearing's outer edge rode against the housing. In such cases, the change in vibration would be detected in a timelier manner through continuous online monitoring.

\subsection{Preventative maintenance recommendations}

Maintenance plans are established to ensure safety and reliability during plant operation. PM is one aspect of such plans, and it is performed at pre-defined intervals, regardless of the component's current 
condition. This is analogous to changing the oil in your car every 5,000 miles without checking the engine condition or oil quality. PM also includes regularly scheduled inspections, testing, and refurbishments. These tasks are implemented in an attempt to reduce the number of failures and downtimes. However, these tasks can be both costly and labor-intensive. For this reason, the frequencies at which PM tasks are performed should be reviewed and updated as more operating experience is acquired. This creates a feedback loop in which maintenance frequency impacts component performance - in turn, affecting maintenance frequency. To speed up this feedback loop, current PM frequencies for healthy components are compared against the recommendations in the EPRI PMBD. In addition to recommended PM frequencies for select tasks, this database includes failure modes and discovery methods. By reviewing a component's operating history and current PM frequency, a justification can be made for suggesting that the frequency be extended. The risk associated with extending the PM task frequency can be also calculated using the EPRI PMBD.

Table 1. Example of current PM frequencies and recommendations.

\begin{tabular}{|l|l|l|l|l|}
\hline \multirow{5}{*}{ Component } & \multicolumn{1}{|c|}{ PM Task } & $\begin{array}{c}\text { Current PM } \\
\text { Frequency }\end{array}$ & \multicolumn{1}{|c|}{ EPRIPMBD } & \multicolumn{1}{|c|}{ Recommendation } \\
\hline CP and CBP & Refurbishment & 8 years & As required & $\begin{array}{l}\text { Good candidate for } \\
\text { frequency extension }\end{array}$ \\
\cline { 2 - 6 } & $\begin{array}{l}\text { Vibration } \\
\text { Monitoring }\end{array}$ & 3 months & 3 months & Keep \\
\cline { 2 - 6 } & Oil Analysis & 6 months & 6 months & Keep \\
\hline & $\begin{array}{l}\text { Vibration } \\
\text { Analysis }\end{array}$ & 3 months & 3 months & Keep \\
\cline { 2 - 6 } & Fan Cleaning & 6 months & 2 months & Keep \\
\cline { 2 - 6 } & \begin{tabular}{l} 
Oil Analysis \\
\cline { 2 - 5 }
\end{tabular} & 6 months & $\begin{array}{l}6 \text { months or } \\
1 \text { year }\end{array}$ & $\begin{array}{l}\text { Good candidate for } \\
\text { frequency extension }\end{array}$ \\
\cline { 2 - 5 } & $\begin{array}{l}\text { Electrical } \\
\text { Testing/ }\end{array}$ & 5 years & 4 years & Keep \\
\hline
\end{tabular}

Over the 5-year period, a total of 705 CBM activities were undertaken between the two PWR systems with regards to the FWCS. These activities vary in location and severity, ranging from simple inspections or alignments to bearing failures and equipment replacements. This report focuses specifically on mechanical issues stemming from the CPs, CBPs, and their respective motors. The CBM work orders were reviewed first, in order to ascertain the historical health of the component.

The CBPs in unit 1 primarily experienced issues concerning mechanical seal leaks. CBP 11 showed three instances of leakage or seal problems. Mechanical seals were also replaced twice: once in 2017 and once in 2018. There was also a possible water intrusion event in 2016, but no other major problems were observed. CBP 12 had only one instance of condensate leak in 2018. CBP 13 had two instances of seal leakage: one in 2016 and one in 2019.

The CPs experienced issues in regard to their connections to the condensate lube oil pump (CLOP). The CLOP for CP 13 experienced several problems, including an oil leak in 2015 and both a coupling 
break and bearing fault frequencies in 2018. Each CLOP in unit 1 was replaced to fix an alignment issue, but no such issues were reported in 2019 or 2020.

Unit 2 experienced fewer leak issues than unit 1. CBP 21 had one instance of leakage, one thrust bearing temperature sensor replacement, and one unplanned stoppage during start-up - with no reason detailed in the work orders. The temperature sensor replacement was most likely a reinstallation of the temperature sensor and was not gapped properly. CBP 22 had no leaks, but did require that a stanchion be replaced to an elbow. CP 23 had two instances of oil leakage: one in 2017 and one in 2019. The CP 21 CLOP saw impacting within vibration readings in 2018. The CP 22 CLOP had an oil leak in 2018, as well as two instances of running while the pump was secured. Though having the CLOP running while the CP is offline is not detrimental to the health of the CP, it could reveal a configuration issue. The CP 23 CLOP reported one instance of elevated noise, and the CLOP was replaced to fix alignment issues, as was also the case with unit 1.

The records of the $\mathrm{CP}$ and $\mathrm{CBP}$ motors for both units 1 and 2 were nearly impeccable. The only recorded issues were dirty filters in the unit 1 motors in 2019. That sort of record can open up opportunities to extend PM frequencies for cost reduction purposes. A summary of PM tasks and the frequencies at which these tasks are performed for each component is given in Table 1. Next to each component's PM frequency is the EPRI-PMBD-recommended frequency based on subject-matter-expert opinion and component reliability data. Among the seven listed PM tasks, four have current frequencies that differ with those recommended by the EPRI PMBD. Based on CM history and the EPRI-PMBDrecommended frequencies, two tasks were deemed good candidates for PM frequency extensions: pump refurbishment and motor oil analysis.

A "refurbishment" is defined as replacing a component with a spare and then sending it off to be overhauled and repaired. Refurbishments are conducted every eight years during a plant outage, thus avoiding any unnecessary downtimes. Tables 2 and 3 list the work orders for component refurbishments for units 1 and 2, respectively. Since this work order history dates back five years, some component refurbishments are not listed, due to the 8-year frequency. Overall, the CPs and CBPs had a positive CM work history. With their EPRI-PMBD-recommended PM frequency of "as required," CP/CBP component refurbishment is a good candidate for PM frequency extension, though the final decision should not be based solely on component performance. An appropriate risk assessment and review of available sensors must be undertaken to determine the health of the pumps. To aid in determining when the pumps should be refurbished, it is recommended that the vibration monitoring and oil analysis continue at their current frequencies. These tasks can be used to monitor component health while trending any observable degradation. Additionally, routine-based vibration monitoring can be replaced with online vibration monitoring (where applicable) in order to further aid in this process. The decision of when to refurbish is a complex one, requiring more information than available for this report.

Table 2. Most recent refurbishment work order for the unit 1 CPs, CBPs, and their associated drive motors.

\begin{tabular}{|l|l|l|l|l|}
\hline \multirow{3}{*}{ NPP 1 } & Unit & 11 & 12 & 13 \\
\cline { 2 - 5 } & $\mathrm{CP}$ & $5 / 27 / 16$ & $7 / 15 / 20$ & $5 / 27 / 16$ \\
\cline { 2 - 5 } & CP Motor & $5 / 27 / 16$ & $7 / 15 / 20$ & $5 / 27 / 16$ \\
\cline { 2 - 5 } & CBP & $\begin{array}{l}10 / 12 / 17 \\
6 / 23 / 20\end{array}$ & N/A & $10 / 12 / 17$ \\
\cline { 2 - 5 } & CBP & N/A & N/A & $\begin{array}{l}5 / 27 / 16 \\
7 / 15 / 20\end{array}$ \\
\cline { 2 - 5 } & Motor & & & \\
\hline
\end{tabular}


Table 3. Most recent refurbishment work order for the unit 2 CPs, CBPs, and their associated drive motors.

\begin{tabular}{|l|l|l|l|l|}
\hline \multirow{4}{*}{ NPP 2 } & Unit & 21 & 22 & 23 \\
\cline { 2 - 5 } & CP & N/A & $4 / 10 / 19$ & $3 / 2 / 17$ \\
\cline { 2 - 5 } & CP Motor & $3 / 2 / 17$ & $4 / 16 / 19$ & $3 / 2 / 17$ \\
\cline { 2 - 5 } & CBP & N/A & N/A & $5 / 27 / 15$ \\
\cline { 2 - 5 } & $\begin{array}{l}\text { CBP } \\
\text { Motor }\end{array}$ & N/A & $4 / 10 / 19$ & $5 / 27 / 15$ \\
\hline
\end{tabular}

The motors driving the CPs and CBPs have experienced minimal problems over the last 5 years. The one instance noted in the CM work orders was due to dirty filters in the unit $1 \mathrm{CP}$ motors. Since the overall health of the components has been good over a significant period of time, PM frequency extensions should be considered in order to reduce the overall cost of maintenance. Since the only issue that the motors faced were dirty filters, and the current PM frequency is already longer than that recommended by the EPRI PMBD, the fan-cleaning frequency should not be adjusted.

There is a potential opportunity to extend the frequencies of vibration and/or oil analyses for the motors. Table 1 lists the EPRI-PMBD-recommended frequencies as being 3 months for the vibration analysis and 6 months or a year for the oil analysis, depending on whether the motor is classified as critical or noncritical, respectively. Either of these frequencies could potentially be extended, perhaps even both. Furthermore, the vibration-monitoring PM task could potentially be replaced by continuous online monitoring, which is why Table 1 suggests oil analysis to be the preferred candidate for PM frequency extension. In the PWR system, all four CPs must be running for the system to operate at 100\% capacity. The motors that drive the CPs are critical components. The CBPs are operated in a two-out-ofthree fashion for $100 \%$ capacity. Their operation is alternated regularly in order to evenly spread out the accumulated wear. In this case, a backup CBP is present in case one fails to operate. Even with a potential backup, the CBP motors may still be considered critical, and a 6-month oil analysis may be advisable. With a risk analysis and more information about the oil analysis contents, the PM frequency could likely be extended to 9 months or even a year.

\section{SUMMARY AND PATH FORWARD}

O\&M costs for NPPs are very large but could potentially be reduced thanks to advances in fault diagnostics and PMO. The CMMS contains work orders detailing maintenance activities, and these can be used to identify and label faults. ML techniques such as SVM, in combination with feature extraction techniques, can be utilized to search for similar instances of those faults. Results indicate that not all faults can be identified via the current suite of embedded sensors. Additional sensors or inspections would be required to locate these elusive faults. This paper also showed the analytical steps necessary for PMO, from component health determinations to recommendations for action. This type of analysis can be extended to components within other systems, as long as their performance histories and PM frequencies are known. The final determination as to which PM tasks to extend (and to what frequencies) should be made in light of both the component's performance history and the results of a risk analysis. Further investigation needs to be made into the cause of anomalies observed within the data but not explained by the CMMS. These anomalies could be due to CMMS bookkeeping errors, operational shifts, sensor drift, etc. 


\section{ACKNOWLEDGEMENTS}

This report was made possible through funding by the U.S. Department of Energy's Office of Nuclear Energy under the Nuclear Energy Enabling Technologies Program. We are grateful to Suibel Schuppner at the U.S. Department of Energy and Craig Primer at Idaho National Laboratory for championing this effort. The data used in this research was provided by Exelon Generation Company. We are also thankful to the plant engineers for their technical discussions on the data.

\section{REFERENCES}

[1] Robert Bryce, "Nuclear Plants In Illinois Are Slated For Closure. Will The State's Democratic Politicians Save Them?" [Online]. Available: https://www.forbes.com/sites/robertbryce/2020/08/31/nuclear-plants-in-illinois-are-slated-forclosure-will-the-states-democratic-politicians-save-them/?sh=5e6cf62511 ef.

[2] Shannon M. Bragg-Sitton, Richard Boardman, Cristian Rabiti, and James O'Brien. "Reimagining future energy systems: Overview of the US program to maximize energy utilization via integrated nuclear-renewable energy systems.” International Journal of Energy Research 44, no. 10 (August 2010): 8156-8169. First published 2020 Feb 17. https://doi.org/10.1002/er.5207

[3] Y. Lei, B. Yang, X. Jiang, F. Jia, N. Li, and A. K. Nandi, "Applications of machine learning to machine fault diagnosis: A review and roadmap," Mech. Syst. Signal Process. 138, p. 106587, 2020, 10.1016/j.ymssp.2019.106587.

[4] Nuclear Energy Institute, "Industry Guideline for Monitoring the Effectiveness of Maintenance At Nuclear Power Plants," NUMARC 93-01 Revision 4A (April 2011). nrc.gov/docs/ML1111/ML11116A198.pdf

[5] V. Agarwal, "Wireless sensor modalities at a nuclear power site to collect vibration data," Idaho National Laboratory, Idaho Falls, ID, May 2020. INL/EXT-20-58548 Rev 0.

[6] K. A. Manjunatha and V. Agarwal, "Techno-economic analysis framework for wireless networks in nuclear power plants," Idaho National Laboratory, Idaho Falls, ID, September 2019.

INL/EXT-19-55830 Rev 0.

[7] V. Agarwal, "Enabling predictive maintenance with wireless instrumentation in balance of plant system," Idaho National Laboratory, Idaho Falls, July 2019. INL/EXT-21-54866 Rev 1.

[8] K. A. Manjunatha and V. Agarwal, "Review of wireless communication technologies and technoeconomic analysis," Idaho National Laboratory, Idaho Falls, ID, May 2019.

INL/EXT-19-53966 Rev 0.

[9] Pradeep Ramuhalli, Cody Walker, Vivek Agarwal, and Nancy Lybeck, "Development of prognostic models using plant asset data," Oak Ridge National Laboratory, Oak Ridge, TN, September 2020.

[10] V. Agarwal, N. Lybeck, B. Pham, R. Rusaw, and R. Bickford, “Asset Fault Signatures for Prognostic and Health Management in the Nuclear Industry," 2014 IEEE International Conference on Prognostics and Health Management. Eastern Washington University. Spokane, WA.

[11] Parekh, K.J., Solanki, S.K.B.B., Vankar, B.B.R.B., Raval, H.D.B. and Joshi, R.M., “Analysis of the Induction Motor used in Temperature Controller Machine by Condition Based Monitoring System.” International Journal of Science Technology \& Engineering (2017) Vol 3 Issue 10. ISSN: 2349-784X. 
[12] EPRI, "Electrical Power Research Institute Preventative Maintenance Basis Database.” June 30, 2015.

[13] Hamed Khorasgani, Ahmed Farahat, Kosta Ristovski, Chetan Gupta, and Gautam Biswas. "A framework for unifying model-based and data-driven fault diagnosis." Annual Conference of the PHM Society, 10, no. 1 (September 24, 2018). https://doi.org/10.36001/phmconf.2018.v10i1.530

[14] Olivier Janssens, Viktor Slavkovikj, Bram Vervisch, Kurt Stockman, Mia Loccufier, Steven Verstockt, Rik Van de Walle, Sofie Van Hoecke. "Convolutional neural network based fault detection for rotating machinery." Journal of Sound and Vibration 377 (2016): 331-345. https://doi.org/10.1016/j.jsv.2016.05.027

[15] Jungwon Yu, Jaeyeong Yoo, Jaeyel Jang, June Ho Park, Sungshin Kim, “A novel hybrid of autoassociative kernel regression and dynamic independent component analysis for fault detection in nonlinear multimode processes.” Journal of Process Control 68 (2018): 129-144.

https://doi.org/10.1016/j.jprocont.2018.05.004

[16] Alberto Diez-Olivana, Javier Del Ser, Diego Galarad, and BasilioSierra, "Data fusion and machine learning for industrial prognosis: Trends and perspectives towards Industry 4.0," Inf. Fusion 50 (2019): 92-111. 10.1016/j.inffus.2018.10.005.

[17] Harris Drucker, Christopher J. C. Burges, Linda Kaufman, Alex Smola, and Vladimir Vapnik. "Support vector regression machines," Advances in Neural Information Processing Systems 28 (1997): 155-161.

[18] B. Dong, C. Cao, and S. E. Lee, "Applying support vector machines to predict building energy consumption in tropical region," Energy Build. 37, no. 5 (2005): 545-553. 10.1016/j.enbuild.2004.09.009.

[19] R. K. Jain, K. M. Smith, P. J. Culligan, and J. E. Taylor, "Forecasting energy consumption of multi-family residential buildings using support vector regression: Investigating the impact of temporal and spatial monitoring granularity on performance accuracy," Appl. Energy 123 (2014): 168-178. 10.1016/j.apenergy.2014.02.057. 